\title{
Problemas difusivos bIDIMENSIONAIS EM REgIME PERMANENTE COM FONTE ARBITRÁRIA - SOLUÇÕES EXATAS
}

\author{
STEADY TWO-DIMENSIONAL DIFFUSION PROBLEMS WITH ARBITRARY \\ SOURCES - EXACT SOLUTIONS
}

\begin{abstract}
JORGE RODOLFO SILVA ZABADAL
Engenheiro Quimico(UFRGS), Mestre em engenharia (PROMEC/UFRGS), Doutor em engenharia (PROMEC/UFRGS), professor adjunto do departamento de engenharia nuclear
\end{abstract}

\section{DANIEL BECK}

Engenheiro Mecânico (UFRGS), Mestre em engenharia (PROMEC/UFRGS)

\section{RENATO LETIZIA GARCIA} Engenheiro Quimico(UFRGS), Mestre em engenharia (IPH/UFRGS), professor do curso de
engenharia em energia (UERGS)

\section{ELENARA CORRÊA LERSCH}

Engenheira Química (UFRGS), Departamento Municipal de Água e Esgotos (DMAE) - Divisão de Pesquisa

\section{CÉSAR XAVIER HOFFMANN}

Engenheiro Químico (UFRGS), Departamento Municipal de Água e Esgotos (DMAE) - Centro de Suporte ao Usuário

$$
\text { Recebido: 19/01/05 Aceito: 28/09/05 }
$$

\section{RESUMO}

Neste trabalho são apresentados métodos híbridos para solução de problemas difusivos relativos à dispersão de poluentes em meio aquático. Estes métodos aplicam variáveis complexas a fim de executar mapeamentos sobre a equação diferencial a ser resolvida bem como sobre o domínio considerado. $\mathrm{O}$ mapeamento sobre a equaçáo diferencial converte o operador laplaciano bidimensional em uma derivada cruzada de segunda ordem na variável espacial. $\mathrm{O}$ mapeamento do domínio transforma regiōes de formato complexo em regiôes retangulares. Ambos mapeamentos são usados a fim de reduzir o tempo total requerido de processamento para solução de problemas difusivos não-homogêneos. Resultados numéricos são apresentados.

PALAVRAS-CHAVE: Problemas difusivos, equação de poisson, variáveis complexas, transformações conformes.

\begin{abstract}
In this work hybrid methods for solving diffusion problems related to pollutants dispersion in water bodies are presented. These methods employ complex variables in order to perform mappings over the differential equation to be solved as well as over the considered domain. The mapping over the differential equation converts the two dimensional laplacian operator into a second order mixed derivative in the complex variables. The mapping of the domain transforms complex-shaped regions into rectangular ones. Both mappings are used in order to reduce the total time proccessing required for solving non-homogeneous diffusion problems. Numerical results are reported.
\end{abstract}

KEYWORDS: Diffusion problems, poisson equation, complex variables, conformal mapping. $\frac{\partial^{2} f}{\partial y^{2}}+\frac{\partial^{2} f}{\partial x^{2}}=Q(x, y)$

onde $\mathrm{Q}(\mathrm{x}, \mathrm{y})$ representa a fonte arbitrária. A equação (1) é também conhecida como equação de Poisson e descreve fenômenos difusivos, tanto em transferência de energia quanto de massa.

Em muitos casos, a geometria de um determinado problema pode dificultar ou até mesmo impedir sua solução analítica devido à aplicação das condições de contorno, sendo necessário na maioria dos casos um tratamento numérico para a questão. $O$ foco central do trabalho consiste em apresentar uma metodologia de solução híbrida para a equação (1), a qual possa ser aplicada nos casos onde a geometria ou condições de contorno dificulte a obtenção da solução analítica do problema. 
$\mathrm{O}$ processo físico relacionado à difusão está diretamente ligado à atividade atômica e molecular do meio no qual o fenômeno está ocorrendo. A difusão, para o caso da transferência de calor, pode ser vista como a transferência de energia de partículas mais energéticas para partículas de menor energia devido às interações entre elas (Incropera \& DeWitt, 1981). Para o caso da transferência de massa, os fenômenos a nível molecular são análogos ao caso da transferência de energia. Sendo assim, o tratamento matemático para estes dois processos físicos, tanto de condução do calor quanto de difusão de massa, são semelhantes.

A dispersão de poluentes em meio aquático ocorre, fundamentalmente, através de dois processos: difusão e advecção. O primeiro, conforme descrito, independe das condiçôes hidrodinâmicas do sistema, salvo a influência de componentes turbulentas, ocorrendo, inclusive, em locais onde não há escoamento ou as velocidades são insignificantes. A advecção consiste na parcela de dispersão produzida pelo transporte através da correnteza do corpo hídrico.

O modelamento matemático proposto neste trabalho diz respeito à equação $\mathrm{da}$ difusão bidimensional em regime permanente, equação (1). Para este tipo de tratamento, existem diversos métodos matemáticos que possibilitam a solução do problema. Dentre os mais utilizados encontramse o método de separação de variáveis (Ozisik, 1994; Ayres, 1985; Boyce, 1995 ) e a análise por diferenças finitas(Carnaham;1969). Existem ainda outras possibilidades de solução, como o Método Gráfico (Incropera \& DeWitt; 1981) ou, para problemas transientes, a aplicação da transformada de Laplace. (Kakaç,Yener,1993)

A limitação do método da separação de variáveis diz respeito à aplicação das condições de contorno, as quais podem inviabilizar a solução do problema. Geralmente esta metodologia só serve para uma gama de geometrias e condições de contorno simples.

Em se tratando de problemas que envolvam geometrias e/ou condições de contorno mais complexas, usualmente utilizam-se métodos de soluçôes numéricos, como equações em Diferenças Finitas e elementos finitos. Ao contrário de uma solução analítica, que permite a determinação da função em qualquer ponto de interesse no interior do domínio, uma análise numérica fornece a solução em pontos discretos, estando a precisão relacionada diretamente com a correta seleção destes pontos, bem como o tamanho das regiões.

\section{METODOLOGIA}

O desenvolvimento proposto consiste em estabelecer uma seqüência de etapas para a obtenção da solução da equação de Poisson. Este trabalho não tem intenção de detalhar todos os teoremas matemáticos envolvidos, mas sim objetivar a aplicação prática dos mesmos.

\section{Equação de Poisson em termos das variáveis complexas}

A equação (1) pode ser escrita em termos das variáveis complexas. A mudança de variável se dá pela introdução da variável complexa $\mathrm{z}$ e seu conjugado $\mathrm{z}$, como segue:

$$
\begin{aligned}
& \mathrm{z}=\mathrm{x}+\mathrm{iy} \\
& \overline{\mathrm{z}}=\mathrm{x}-\mathrm{iy}
\end{aligned}
$$

Pela aplicação da regra da cadeia na equação de Poisson, temos

$$
\begin{aligned}
& \frac{\partial f}{\partial x}=\frac{\partial f}{\partial z} \frac{\partial z}{\partial x}+\frac{\partial f}{\partial \bar{z}} \frac{\partial \bar{z}}{\partial x}=\frac{\partial f}{\partial z}+\frac{\partial f}{\partial \bar{z}}=g \\
& \frac{\partial^{2} f}{\partial x^{2}}=\frac{\partial g}{\partial z}+\frac{\partial g}{\partial \bar{z}}=2 \frac{\partial^{2} f}{\partial z \partial \bar{z}}+\frac{\partial^{2} f}{\partial z^{2}}+\frac{\partial^{2} f}{\partial \bar{z}^{2}}
\end{aligned}
$$

Usando a mesma sistemática para a diferencial com relação a y, temos:

$\frac{\partial^{2} \mathrm{f}}{\partial \mathrm{y}^{2}}=2 \frac{\partial^{2} \mathrm{f}}{\partial \mathrm{z} \partial \overline{\mathrm{z}}}-\frac{\partial^{2} \mathrm{f}}{\partial \mathrm{z}^{2}}-\frac{\partial^{2} \mathrm{f}}{\partial \overline{\mathrm{z}}^{2}}$

Substituindo a equação (3) e (4) na equação de Laplace, resulta

$$
\nabla^{2} \mathrm{f}=\frac{\partial^{2} \mathrm{f}}{\partial \mathrm{x}^{2}}+\frac{\partial^{2} \mathrm{f}}{\partial \mathrm{y}^{2}}=4 \frac{\partial^{2} \mathrm{f}}{\partial \mathrm{z} \partial \overline{\mathrm{z}}}
$$

Neste ponto pode-se verificar a utilidade da representação nestes termos, visto que a solução do Laplaciano pode ser alcançada diretamente através de uma integração dupla nas novas variáveis.

Uma vez estabelecido o Laplaciano em termos de $\mathrm{Z}$ e $\bar{Z}$, a etapa seguinte é realizar a mudança de variável na fonte
$\mathrm{Q}(\mathrm{x}, \mathrm{y})$. Como z e seu conjugado $\overline{\mathrm{z}}$ são funçôes das variáveis reais $\mathrm{x}$ e $\mathrm{y}$, para que a mudança de variável seja feita, basta isolar as variáveis da equação (2), a fim de obter a relação de x e y como função de $z$ e $\bar{Z}$. Assim,

$x=\frac{z+\bar{z}}{2}$
$y=\frac{i(\bar{z}-z)}{2}$

Realizada esta etapa, a equação de Poisson pode ser então expressa em termos das variáveis complexas da seguinte forma:

$\nabla^{2} \mathrm{f}=4 \frac{\partial^{2} \mathrm{f}}{\partial \mathrm{z} \partial \overline{\mathrm{z}}}=\mathrm{q}(\mathrm{z}, \overline{\mathrm{z}})$

Com a equação (7) estabelece-se uma solução imediata, a qual pode ser alcançada simplesmente por dupla integração do termo fonte $q(z, \bar{z})$, somando ao espaço nulo do operador,

$\mathrm{f}=\frac{1}{4} \iint \mathrm{q}(\mathrm{z}, \overline{\mathrm{z}}) \mathrm{dzd} \overline{\mathrm{z}}+\mathrm{f}_{1}(\overline{\mathrm{z}})+\mathrm{f}_{2}(\mathrm{z})$

Nesta equação, $f_{1}(\bar{z})+f_{2}(z)$ correspondem ao espaço nulo do operador Laplaciano, pois $\nabla^{2}\left(\mathrm{f}_{1}(\overline{\mathrm{z}})+\mathrm{f}_{2}(\mathrm{z})\right)=0$. Para casos em que a geometria do domínio não represente um problema para a aplicação das condições de contorno, a expressão (8) é a solução final do problema, sendo aplicada diretamente no domínio original, ou seja, sem a utilização das transformações conformes.

\section{Transformações conformes}

A aplicação do método das transformaçôes conformes consiste em um mapeamento de pontos entre dois domínios distintos. Seu objetivo é transformar a geometria do domínio original em uma outra geometria de maior simplicidade.

Para exemplificar o método, vejamos o caso da função $\mathrm{f}(\mathrm{z})=\mathrm{z}^{2}$, a qual pode ser expressa em função das coordenadas cartesianas $(\mathrm{x}, \mathrm{y})$.

Assim, $\mathrm{z}=\mathrm{x}+\mathrm{iy}$, e substituindo na equação original, temos

$$
\begin{aligned}
& f(z)=\left(x+i y^{2}\right)=x^{2}-y^{2}+i(2 x y)= \\
& =u(x, y)+i v(x, y)
\end{aligned}
$$

e que pode ser expressa em termos das variáveis u e v. 
Define-se então um ponto arbitrário no plano w como sendo

$\mathrm{w}=\mathrm{u}(\mathrm{x}, \mathrm{y})+\mathrm{iv}(\mathrm{x}, \mathrm{y})$

Tomando-se uma reta horizontal no plano z, ou seja, y=cte (figura. 1) e aplicando a transformação acima descrita, obtêm-se uma nova geometria no plano w (figura. 2).

As figuras 1 e 2 exemplificam a aplicação de uma transformação conforme. Aplicando a transformação correspondente a $f(z)=z^{2}$ na reta da figura 1, gera-se uma nova geometria, correspondente à figura 2 . Note-se que o processo pode ser revertido, aplicando-se a transformação inversa, ou seja, $f(w)=\sqrt{w}$.

Segundo Dettman (1965), para que uma transformação possa ser conforme, existem dois requisitos que precisam ser satisfeitos:

$1^{\circ}$ - A função $f(z)$ aplicada no plano $\mathrm{z}$ precisa ser analítica no domínio.

$2^{o}$ - A derivada da função $\mathrm{f}(\mathrm{z})$ não pode ser nula, i.e., $\mathrm{f}^{\prime}(\mathrm{z}) \neq 0$

Qualquer transformação que satisfaça estes dois requisitos é dita conforme.

A principal característica deste tipo de transformação é o fato de preservar a estrutura da equação de Laplace. Isto significa que não importa qual a função complexa aplicada na transformação, ela sempre vai manter a equação inalterada, exceto pela mudança de variáveis. $\mathrm{O}$ resultado obtido com a aplicação de uma função arbitraria sobre a equação de Laplace tem o seguinte formato:

$\left(\frac{\partial^{2} f}{\partial x^{2}}+\frac{\partial^{2} f}{\partial x^{2}}\right)=\left(\frac{\partial^{2} f}{\partial u^{2}}+\frac{\partial^{2} f}{\partial v^{2}}\right)\left(f^{\prime}(z)\right)^{2}=0$

deixando claro o motivo pelo qual a função aplicada não pode ter derivada nula.

\section{Condições de Cauchy- Riemann}

Outra característica de uma transformação conforme é o fato de ela preservar os ângulos de um elemento de área frente a uma transformação. Isto significa que a malha de um determinado domínio preserva seus ângulos quando mapeada em outro domínio. Segundo Dettman (1965), esta propriedade decorre diretamente das condiçôes de Cauchy-Riemann: tomando $\mathrm{z}=\mathrm{x}+\mathrm{iy}$,

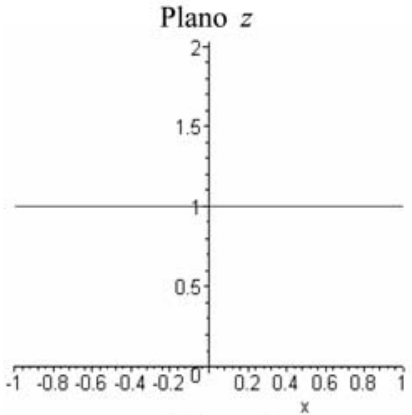

(Figura 1)

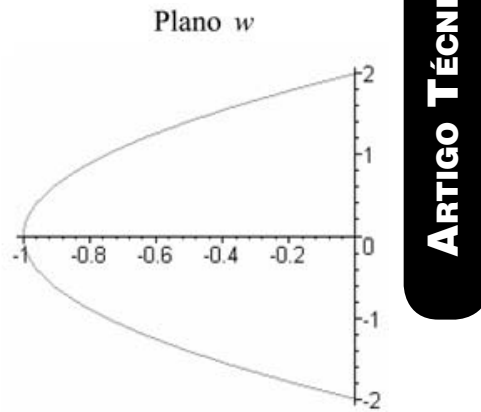

(Figura 2)
Figuras I e 2 - Transformação conforme do plano z para o plano w qualquer função desta variável complexa pode ser expressa como $\mathrm{f}(\mathrm{z})=\mathrm{u}(\mathrm{x}, \mathrm{y})+$ + iv $(x, y)$. A derivada desta função está representada na equação 12 .

Para que $\mathrm{f}(\mathrm{z})$ seja analítica, este limite deve existir independentemente de como $\Delta z$ tenda para zero, seja pela aproximação por $\mathrm{x}$ ou pela aproximação por y. Assim, fazendo $\Delta y$ tender a zero antes de $\Delta x$, tem-se a equação 13 .

Por outro lado, fazendo $\Delta \mathrm{x}$ tender a zero antes de $\Delta y$, obtêm-se a equação 14 .

Para que $f(z)$ seja analítica, $f^{\prime}(z)$ deve ser única, assim $\mathrm{f}_{1}{ }^{\prime}(\mathrm{z})=\mathrm{f}_{2}{ }^{\prime}(\mathrm{z})$. Isto implica nas equaçôes 15 e 16 .

Estas equações são conhecidas como condições de Cauchy-Riemann e necessariamente precisam ser respeitadas em qualquer função de variável complexa. Estas condições também garantem a ortogonalidade entre as isolinhas de uma função de variável complexa.

Tendo a garantia da invariância da equação e a garantia da invariância dos ângulos da malha pelas condições de Cauchy-Riemann, a transformação conforme pode ser então aplicada ao problema.

\section{Paramétricas que mapeiam o domínio}

A descrição das transformações conformes vista na seção anterior se refere aos casos onde a função aplicada na transformação é conhecida, ou seja, aplica-se a função no domínio original de forma a obter o domínio transformado. Porém, para problemas práticos em engenharia, é necessário estabelecer uma forma de determinar qual a função que faz o mapeamento entre dois domínios já conhecidos. Para que este tipo de abordagem possa ser realizado, é necessária a aplicação das funções paramétricas, as quais são responsáveis pelo mapeamento entre os planos.

As paramétricas que mapeiam o domínio podem ser vistas como a função pela qual se dará a transformação

$$
\begin{aligned}
& \mathrm{f}^{\prime}(\mathrm{z})=\lim _{\Delta \mathrm{z} \rightarrow 0} \frac{\mathrm{f}(\mathrm{z}+\Delta \mathrm{z})-\mathrm{f}(\mathrm{z})}{\Delta \mathrm{z}}= \\
& =\lim _{\substack{\Delta \mathrm{z} \rightarrow 0 \\
\Delta \mathrm{y} \rightarrow 0}} \frac{\mathrm{u}(\mathrm{x}+\Delta \mathrm{x}, \mathrm{y}+\Delta \mathrm{y})+\mathrm{iv}(\mathrm{x}+\Delta \mathrm{x}, \mathrm{y}+\Delta \mathrm{y})-(\mathrm{u}(\mathrm{x}, \mathrm{y})+\mathrm{iv}(\mathrm{x}, \mathrm{y}))}{\Delta \mathrm{x}+\Delta \mathrm{y}} \\
& \mathrm{f}_{1}{ }^{\prime}(\mathrm{z})=\lim _{\Delta \mathrm{x} \rightarrow 0} \frac{\mathrm{u}(\mathrm{x}+\Delta \mathrm{x}, \mathrm{y})-\mathrm{u}(\mathrm{x}, \mathrm{y})+\mathrm{i}[\mathrm{v}(\mathrm{x}+\Delta \mathrm{x}, \mathrm{y})-\mathrm{v}(\mathrm{x}, \mathrm{y})]}{\Delta \mathrm{x}}= \\
& =\frac{\partial \mathrm{u}}{\partial \mathrm{x}}+\mathrm{i} \frac{\partial \mathrm{v}}{\partial \mathrm{x}}
\end{aligned}
$$

$$
\begin{aligned}
& f_{2}^{\prime}(z)=\lim _{\Delta y \rightarrow 0} \frac{u(x, y+\Delta y)-u(x, y)+i[v(x, y+\Delta y)-v(x, y)]}{i \Delta y}= \\
& =-i \frac{\partial u}{\partial y}+\frac{\partial v}{\partial y} \\
& \frac{\partial u}{\partial x}=\frac{\partial v}{\partial y} \\
& \frac{\partial u}{\partial y}=-\frac{\partial v}{\partial x}
\end{aligned}
$$


conforme. Esta etapa do processo pode exigir tratamento numérico, consistindo no ajuste de curva dos pontos do domínio original.

A transformação conforme realizada neste trabalho consiste em transformar o domínio original de um determinado problema em um semiplano y positivo. Isto significa que independentemente do formato do domínio original, este sempre poderá ser transformado em um semi-plano y positivo.

As paramétricas podem ser determinadas percorrendo-se o contorno do domínio original e relacionando-os com os pontos desejados no domínio transformado (figura 3).

Para que o levantamento das paramétricas seja efetuado, é necessário um mapeamento entre os pontos do contorno nos plano z e plano w. Para isso utiliza-se o parâmetro $\mathrm{u}$, a fim de determinar a função que correlaciona os pontos de um contorno no outro. $\mathrm{O}$ contorno do domínio original é transformado em uma reta no plano $\mathrm{w}$, a qual coincide com o eixo das abscissas. Gerase então uma tabela para o levantamento das paramétricas, correlacionando as variáveis x e y com o parâmetro u.

A Tabela 1 tem caráter unicamente representativo, objetivando demonstrar o processo de obtenção das funções de ajuste.

Tabela I- Ajuste das curvas das paramétricas

\begin{tabular}{ccc}
\hline $\mathrm{U}$ & $\mathrm{x}$ & $\mathrm{Y}$ \\
\hline $\mathrm{u}_{1}$ & $\mathrm{x}_{1}$ & $\mathrm{y}_{1}$ \\
$\mathrm{u}_{2}$ & $\mathrm{x}_{2}$ & $\mathrm{y}_{2}$ \\
$\ldots$ & $\cdots$ & $\cdots$ \\
$\mathrm{U}_{\mathrm{m}}$ & $\mathrm{x}_{\mathrm{n}}$ & $\mathrm{y}_{\mathrm{n}}$ \\
\hline
\end{tabular}

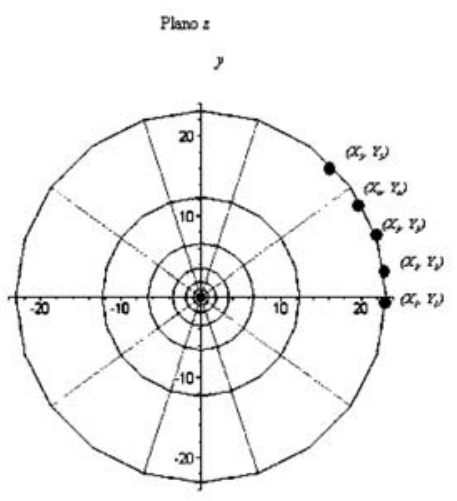

Pelo ajuste de curva surge então duas funções dependentes da variável u,

$\mathrm{X}=\mathrm{X}(\mathrm{u}) \mathrm{e}$

$\mathrm{Y}=\mathrm{Y}(\mathrm{u})$

Para a determinação das paramétricas no interior do domínio, basta fazer a substituição da variável u por $\mathrm{u}+\mathrm{iv}$, a fim de garantir que as funções ajustadas respeitem as condiçōes de Cauchy-Riemann. Assim,

$\mathrm{X}=\mathrm{X}(\mathrm{u}+\mathrm{iv}) \mathrm{e}$

$Y=Y(u+i v)$.

As funções paramétricas devem ser utilizadas após a determinação da solução da equação de Poisson no plano transformado, quando for efetuado o caminho inverso. Essa operação é efetuada por via numérica.

\section{Solução da equação de Poisson}

Antes de iniciar esta seção, é necessário um esclarecimento: $\mathrm{O}$ desenvolvimento da Equação de Poison elaborado no início deste trabalho, explica como é realizada a mudança de variável na equação de Poisson e está desenvolvida para o plano z, porém esta sistemática pode ser utilizada para qualquer plano. Assim, para o plano w temos:

$\mathrm{w}=\mathrm{u}+\mathrm{iv}$

$\overline{\mathrm{W}}=\mathrm{u}-\mathrm{iv}$

e a equação (5) fica com o seguinte formato:

$\nabla^{2} \mathrm{f}=4 \frac{\partial^{2} \mathrm{f}}{\partial \mathrm{w} \partial \overline{\mathrm{w}}}=q(\mathrm{w}, \overline{\mathrm{w}})$

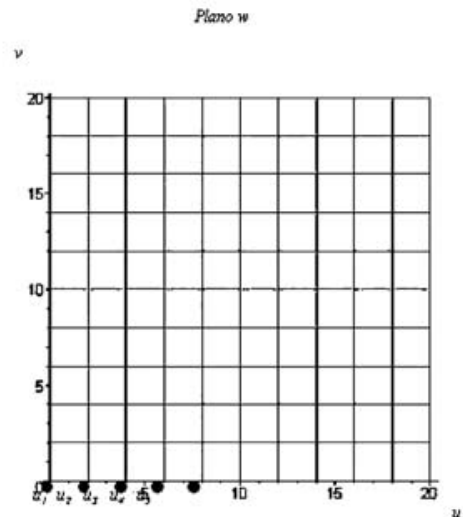

Figura 3 - Ajuste das Paramétricas
O passo a seguir consiste em resolver a equação (20), cuja solução é

$\mathrm{f}=\frac{1}{4} \iint \mathrm{q}(\mathrm{w}, \overline{\mathrm{w}}) \mathrm{dwd} \overline{\mathrm{w}}+\mathrm{f}_{1}(\overline{\mathrm{w}})+\mathrm{f}_{2}(\mathrm{w})$

onde $f_{1}(\bar{w})$ e $f_{2}(w)$ representam o espaço nulo do operador.

A equação (21) mostra que a solução para a equação de Poisson pode ser alcançada por integração direta do termo fonte, somada ao espaço nulo do operador.

A solução apresentada depende das variáveis $\mathrm{w}$ e $\overline{\mathrm{W}}$, u seja, $\mathrm{f}=\mathrm{f}(\mathrm{u}+\mathrm{iv}, \mathrm{u}-\mathrm{iv})$. Como esta solução está generalizada em termos das variáveis complexas, é necessário que se faça a particularização para uma função real, visto que para a aplicação proposta neste trabalho, a parte imaginaria não consiste em solução para o problema. Assim,

$\mathrm{f}_{\text {dif }}=\operatorname{Re}(\mathrm{f}(\mathrm{u}+\mathrm{iv}, \mathrm{u}-\mathrm{iv}))$

Porém deve-se ressaltar que esta particularização depende do problema estudado, e não é regra geral de aplicação. Por exemplo, para o caso de problemas de eletrostática relacionados com a Teoria do Potencial, esta particularização não pode ser efetuada (Churchill,1975; Spiegel,1965).

\section{Aplicação das condições de contorno}

A aplicação das condiçôes de contorno é efetuada também no plano, podendo ser condições de primeira, segunda ou terceira espécie.

Para condiçōes de primeira espécie (condição de Dirichlet), onde o valor da função é prescrito na fronteira, basta mapear diretamente os pontos do plano $\mathrm{z}$ para o plano $\mathrm{w}$, mantendo o valor prescrito da função em cada ponto.

Para condições de segunda espécie (condição de Neumann), onde a derivada da função é constante, condiçōes do tipo $-\frac{\partial \mathrm{f}}{\partial \eta}=\mathrm{C}$ são transformadas $\mathrm{em}-\frac{\partial \mathrm{f}}{\partial \mathrm{v}}=\mathrm{C}$ para $\mathrm{v}=0$.

Um caso especial da condição de segunda espécie diz respeito à superfície isolada (para o caso de transferência de calor, adiabática) no qual a derivada da função é nula, resultando em $\frac{\partial \mathrm{f}}{\partial \mathrm{v}}=0$ para $\mathrm{v}=0$. 
Para condições de terceira espécie (condição de Robin), onde a derivada da função é proporcional ao valor da própria função, condiçôes do tipo $-\frac{\partial f}{\partial \eta}=\mathrm{kf}$ são transformadas em $-\frac{\partial \mathrm{f}}{\partial \mathrm{v}}=\mathrm{kf}$ para $\mathrm{v}=0$, onde $\mathrm{k}=$ cte.

Assim, tendo as condições de contorno determinadas em relação ao plano $w$, é possível aplicá-las à equação (22) e, juntamente com as paramétricas, é possível então efetuar o caminho inverso, voltando ao plano z. Note-se que, qualquer que seja a geometria do domínio original, as condições de contorno transformadas são sempre aplicadas sobre uma interface horizontal.

\section{APLICACÃO DO MÉTODỎ}

A aplicação do método descrito até aqui descreve um cenário real de dispersão de poluentes em meio aquático, de grande interesse em Engenharia Ambiental. A análise física do problema é apresentada no decorrer do desenvolvimento, com posterior discussão dos resultados. O problema proposto diz respeito à dispersão de poluentes na bacia hidrográfica do Guaíba (figura 4).

Em certas regiōes distantes do canal de navegaçáo os termos advectivos da equação que rege a dispersão de poluentes podem ser desprezados, de modo que a equação de Poisson pode ser utilizada sem perda apreciável de exatidão para simular o processo de dispersão de poluentes. Duas regiōes típicas no estuário do lago Guaíba onde os termos advectivos podem ser negligenciados são o Lami e a Barra do Ribeiro, mostrados na figura 4. No trabalho proposto os métodos descritos nas seções anteriores serão utilizados para resolver um problema difusivo bidimensional não-homogêneo nas vizinhanças da praia do Lami. Neste caso o termo de fonte representa um arroio onde são lançadas cargas de esgoto doméstico. A distribuição de concentraçôes na foz do arroio Lami foi ajustada a uma função racional empregando o critério dos mínimos quadrados. A função ajustada é dada pela equação (23), onde os coeficientes $a, b$ e $c$ são dados por:

$$
\begin{aligned}
& \mathrm{a}=0,00000012 \\
& \mathrm{~b}=0,0000000097 \\
& \mathrm{c}=0,000137
\end{aligned}
$$

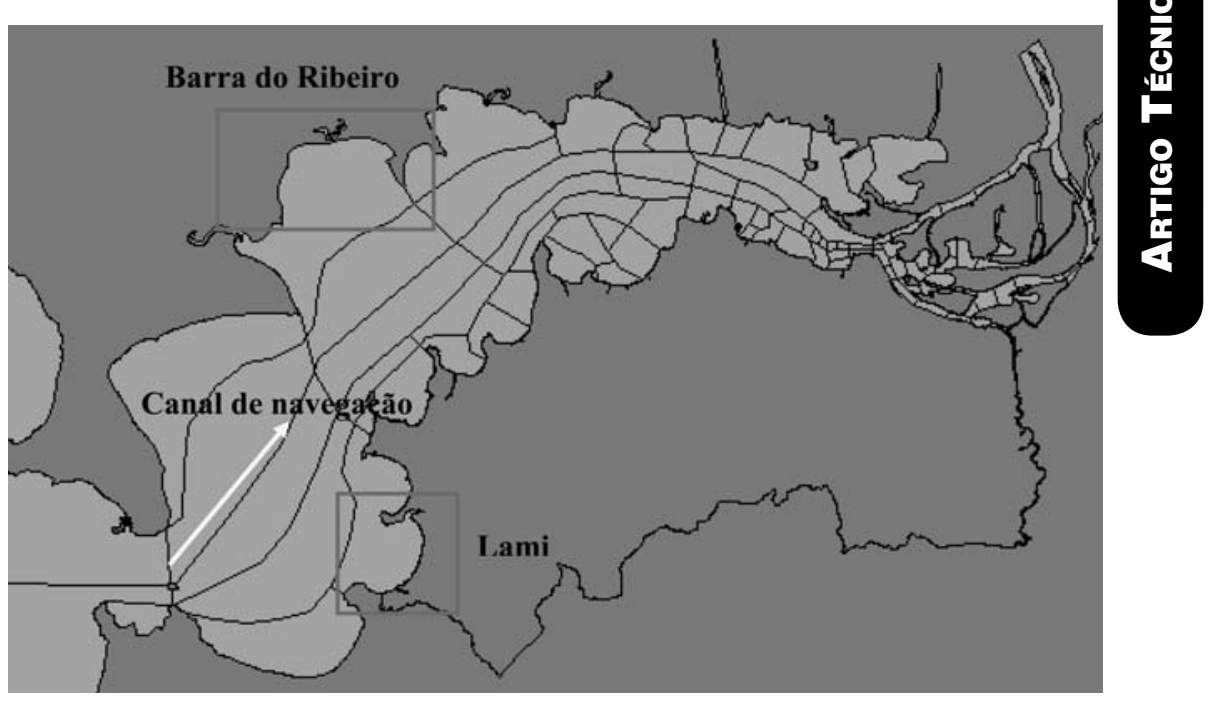

Figura 4 - Estuário do Lago Guaíba

O termo fonte expresso pela equação (23) deve ser representado em termos do plano tranformado, plano $w$. Para isso efetua-se a mudança de variável detalhada na equação (5). a qual em termos do plano transformado toma a forma das equações (24) e (25):

Assim, a equação (23) pode ser escrita na forma da equação (26):

Utilizando o mapeamento descrito anteriormente e integrando a equação resultante, obtem-se a distribuição de concentraçōes no plano $w$, conforme a equação (27).

O gráfico da distribuição no plano $w$ é mostrado na figura 5.

A fim de obter a solução nas variáveis originais, é efetuada uma transformação conforme obtida a partir das equaçōes paramétricas apresentadas abaixo, as quais são obtidas via ajuste de curva com polinômio de quarto grau apresentada nas equaçóes (28) e (29), seguindo a metodologia apresentada anteriormente.
A exemplo da função fonte, as equaçōes paramétricas foram ajustadas a partir de coordenadas geo-referenciadas correspondentes a oito pontos tomados ao longo da praia do Lami (figura 6) utilizando o critério dos mínimos quadrados.

As coordenadas dos pontos amostrados são listadas na Tabela 2, e na figura 7 são representados tais pontos e a respectiva curva paramétrica.

A transformação conforme obtida a partir das paramétricas é dada pela equação (30), onde as coordenadas $x$ e $y$ no sistema de coordenadas original correspon-dem, respectivamente, as partes real e imaginária desta expressão.

A tabela 3 contém alguns pontos de amostragem no interior do domínio e os respectivos valores de concentração de coliformes fecais.

$$
\begin{aligned}
& \mathrm{Q}=\frac{-2\left(-3 \mathrm{a}^{2} \mathrm{u}^{2}+a b v^{2}+a c-3 b^{2} \mathrm{v}^{2}+a b u^{2}+b c\right)}{\left(a u^{2}+b v^{2}+b c\right)^{3}} \\
& \mathrm{u}=\frac{\mathrm{w}+\overline{\mathrm{w}}}{2} \\
& \mathrm{v}=\frac{\mathrm{i}(\overline{\mathrm{w}}-\mathrm{w})}{2} \\
& \mathrm{q}=2 \frac{3 \mathrm{a}^{2}\left(\frac{1}{2} \mathrm{w}+\frac{1}{2} \overline{\mathrm{w}}\right)^{2}+\frac{1}{2} \mathrm{ab}(\overline{\mathrm{w}}-\mathrm{w})^{2}-\mathrm{ac}-\frac{3}{4} \mathrm{~b}^{2}(\overline{\mathrm{w}}-\mathrm{w})^{2}-\mathrm{ab}\left(\frac{1}{2} \mathrm{w}+\frac{1}{2} \overline{\mathrm{w}}\right)^{2}-\mathrm{bc}}{\mathrm{a}\left(\frac{1}{2} \mathrm{w}+\frac{1}{2} \overline{\mathrm{w}}\right)^{2}-\frac{1}{4} \mathrm{~b}(\overline{\mathrm{w}}-\mathrm{w})^{2}+\mathrm{c}} \\
& \mathrm{C}=\frac{1}{0.12 \cdot 10^{-6} \mathrm{u}^{2}+0.97 \cdot 10^{-7} \mathrm{v}^{2}+0.00173}
\end{aligned}
$$


$X=0.8993454402 \cdot 10^{-8} u^{4}+0.7680300298 \cdot 10^{-5} u^{3}+0.000726785969 u^{2}-0.7180652654 u-12.54574145$

$$
\begin{aligned}
\mathrm{z}:= & 0.899345440210^{-8} \mathrm{u}^{4}-0.539607264110^{-7} \mathrm{u}^{2} \mathrm{v}^{2}+0.899345440210^{-8} \mathrm{v}^{4}+ \\
& +0.768030029810^{-5} \mathrm{u}^{3}-0.00002304090089 u v^{2}+0.0007267859693 \mathrm{u}^{2}- \\
& -0.0007267859693 v^{2}-0.7180652654 \mathrm{u}-12.54574145+0.899962656810^{-8} \mathrm{u}^{3} \mathrm{v}- \\
& -0.899962656810^{-8} u v^{3}+0.252400516610^{-5} \mathrm{u}^{2} v-0.841335055510^{-6} v^{3}- \\
& -0.001706353273 u v-1.743903924 v+\left(0.359738176110^{-7} u^{3} v-\right. \\
& -0.359738176110^{-7} u v^{3}+0.00002304090089 u^{2} v-0.768030029810^{-5} v^{3}+ \\
& +0.001453571939 u v-0.7180652654 v-0.224990664210^{-8} u^{4}+ \\
& +0.134994398510^{-7} u^{2} v^{2}-0.224990664210^{-8} v^{4}-0.841335055510^{-6} u^{3}+ \\
& +0.252400516610^{-5} u v^{2}+0.0008531766366 u^{2}-0.0008531766366 v^{2}+ \\
& +1.743903924 u-37.84920304) I
\end{aligned}
$$

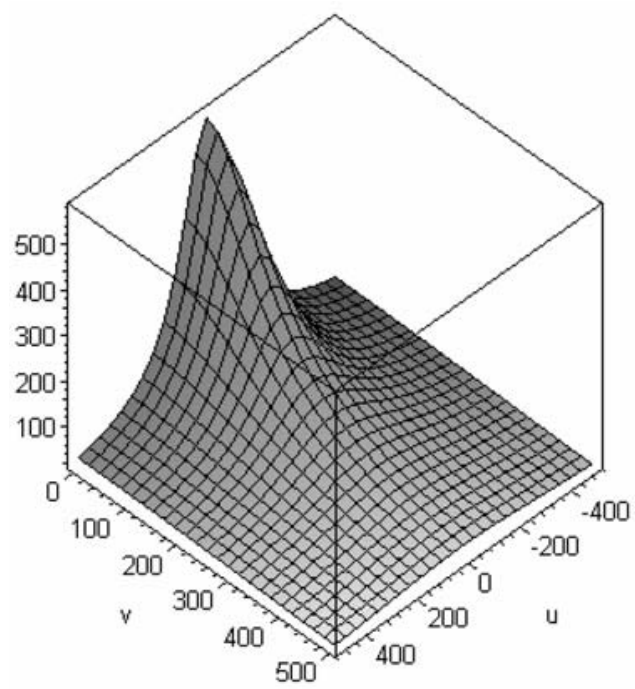

Figura 5 - Dispersão de Poluentes no plano w

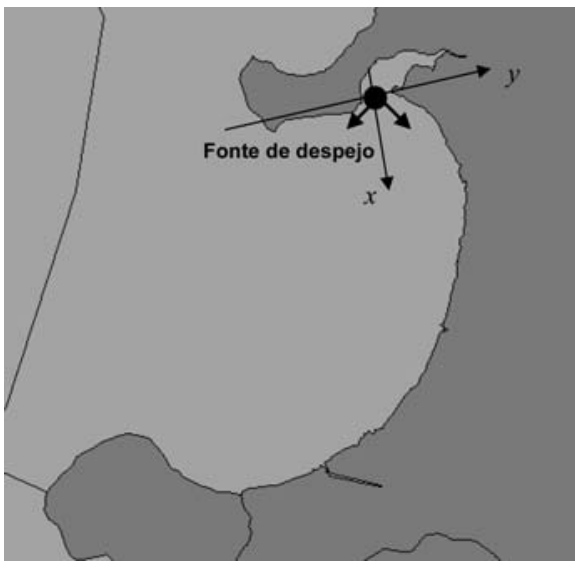

Figura 6 - Sistema de coordenadas para o ajuste das paramétricas

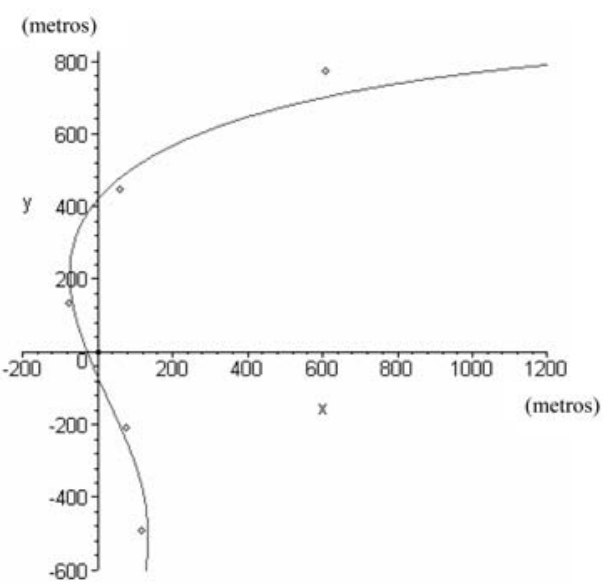

Tabela 2 - Coordenadas dos pontos amostrados

\begin{tabular}{cc}
\hline $\mathrm{X}(\mathrm{m})$ & $\mathrm{Y}(\mathrm{m})$ \\
\hline 120 & -490 \\
80 & -210 \\
-82 & 130 \\
60 & 450 \\
590 & 780 \\
\hline
\end{tabular}

Figura 7 - Pontos de referência para ajuste e curva paramétrica resultante 
Tabela 3 - Valores de concentração de coliformes fecais

\begin{tabular}{cccc}
\hline $\mathrm{X}(\mathrm{m})$ & $\mathrm{Y}(\mathrm{m})$ & $\begin{array}{c}\text { C (calculado) } \\
\text { No coliformes } / 100 \mathrm{~mL}\end{array}$ & $\begin{array}{c}\text { C (dados coletados) } \\
\text { No coliformes } / 100 \mathrm{~mL}\end{array}$ \\
\hline 184.7814997 & -887.9708192 & 22.25679593 & Entre 24 e 30 \\
-717.0198940 & -621.2185510 & 18.61563109 & Entre 24 e 30 \\
132.5169518 & -479.7948214 & 79.80784187 & Entre 70 e 93 \\
3.0853993 & -464.7509432 & 46.90806212 & - \\
-14.29037299 & -38.56812916 & 578.0022739 & Entre 400 e 760 \\
-552.4291822 & -559.2026648 & 95.07242475 & - \\
115.8623462 & 523.9316622 & 79.80784187 & Entre 70 e 93 \\
-1574.678967 & 931.9762258 & 46.90806212 & - \\
2642.805464 & 858.54511060 & 22.25679593 & Entre 24 e 30 \\
-506.4517490 & 5407.953818 & 18.61563109 & Entre 24 e 30
\end{tabular}

\section{DISCUSSÃO}

A tabela 3 mostra os valores estimados para a concentração de coliformes fecais nas proximidades do Arroio Lami, cuja foz se situa no local escolhido como centro do sistema de coordenadas indicado na figura 6. Os valores calculados são consistentes com dados de coleta efetuadas pelo DMAE em campanhas realizadas entre março de 1995 e dezembro de 2003 nas vizinhanças dos pontos amostrados. Note-se que os valores calculados são mais próximos dos limites inferiores dos intervalos dentro dos quais se situam as medidas experimentais. Esse fato decorre, possivelmente, da existência de cargas distribuídas ao longo da região, que não foram consideradas na formulação do problema. Essas cargas correspondem a sistemas de fossas sépticas, infiltraçōes e lençóis subterrâneos cuja presença na área foi detectada, mas que não foram ainda devidamente localizados e registrados para fins de inclusão no modelo como condições de contorno prescritas. Cabe observar que as condições de contorno são consideradas homogêneas, uma vez que toda a carga de esgoto doméstico foi atribuída ao termo de fonte.

Quanto à performance computacional, o sistema correspondente requer cerca de 15 segundos de tempo de processamento, incluindo a plotagem dos gráficos (Celeron - 1GHz, 64Mb RAM). O código fonte, redigido em Maple é extremamente reduzido e de fácil depuração, possuindo cerca de 30 linhas de comando, e empregando apenas recursos básicos do respectivo kernel. O código fonte está descrito no apêndice.

\section{CONCLUSÕES}

Este trabalho mostrou uma metodologia de solução para problemas difusivos voltada para aplicaçóes em problemas de engenharia. Um ponto a ser destacado é a etapa correspondente ao ajuste de curvas para a determinação da função que produz a transformação. Esta etapa possibilita uma maior liberdade para a aplicação da transformação conforme, a qual deixa de ficar atrelada a formas geométricas já conhecidas. Outro ponto de destaque refere-se ao mapeamento da equação de Laplace em termos das variáveis complexas, que transforma a equação original em uma equação de segunda ordem com derivada cruzada, o que facilita a solução da mesma. Dessa forma, a metodologia de solução apresentada pode ser aplicada a inúmeros problemas difusivos que respeitem a equação de Poisson, tornando-se uma ferramenta de grande utilidade para solução de problemas desta natureza.

O tempo de processamento do código computacional resultante possibilita a simulação de cenários em tempo real e, conseqüentemente, viabiliza o seu emprego como ferramenta de gestão ambiental.

O modelamento matemático utilizado pode ser ampliado para simulação de cenários físicos em que as velocidades são significativas e, conseqüientemente, os termos advectivos devem ser considerados na equação. Para tanto basta incluí-los no termo de fonte e aplicar um método iterativo de resolução baseado nos algoritmos de Adomiam (1988). Dessa maneira, as aplicaçôes potenciais do método proposto podem ser estendidas a uma ampla classe de problemas de grande interesse em engenharia ambiental, como, por exemplo, no planejamento de redes de esgoto em municípios banhados por cursos de água que servem simultaneamente para o despejo de efluentes e captação de água para abastecimento. Nesses casos, a utilização de um sistema de simulação de cenários de qualidade de água tornaria possível avaliar o impacto ambiental produzido pelos despejos sobre os balneários utilizados por poluições ribeirinhas e junto aos pontos de captação de água.

\section{REFÊRENCIAS}

ADOMIAM, G. A Review of Decomposition Method in Applied Mathematics. Journal of mathematical analysis and Aplications, n. 235, p. 501-544, 1988.

ÁVILA, G. Variáveis complexas e aplicaçôes. Rio de Janeiro. Livros Técnicos e Científicos Editora, 1990

AYRES, JR. F. Equações Diferenciais. Coleção Schaum. $2^{\circ}$ ed. São Paulo. McGraw-Hill do Brasil, 1985

BOYCE, W. E. Equaçōes Diferenciais Elementares e Problemas de Valores de Contorno. $6^{\circ}$ ed. São Paulo. McGraw-Hill do Brasil, 1995.

CARNAHAM, B. Applied Numerical Methods. New York. John Wiley \& Sons Inc., 604 p, 1969.

CHURCHILL, R. V. Variáveis complexas e suas aplicaçōes. São Paulo. Editora McGraw-Hill do Brasil, 1975.

DETTMAN, J. W. Applied Complex Variables. New York. The Macmillan Company, 1965. 
FIGUEIREDO, D. G. Teoria Clássica do Potencial. Editora Universidade de Brasília, 1963.

INCROPERA, F. P.; DeWITT, D. P. Fundamentos de Transferencia de Calor e de Massa. Rio de Janeiro. Livros Técnicos e Científicos Editora, 1981.

KAKAC, S. Heat Conduction. Taylor \& Francis Publishers, 1993.

NEHARI, Z. Conformal Mapping. $1^{\circ}$ ed. New York. McGraw-Hill Book Company, 396 p, 1952.

OZISIK, M. N. Finite difference methods in heat transfer. CRC Press, 416 p, 1994.

SPIEGEL, M. R. Theory and problems of complex variables, with an introduction to Conformal Mapping and its applications. Schaum's outline series. New York. McGraw-Hill Book company, 1964.
Endereço para correspondência:

\section{Jorge Rodolfo Silva Zabadal \\ Departamento de Engenharia \\ Nuclear - \\ Universidade Federal do Rio \\ Grande do Sul - UFRGS}

Rua Osvaldo Aranha, 86/4 ${ }^{\circ}$ andar

90035-190 Rio Grande do Sul - RS

- Brasil

Tel.: (5I)3316-3327

E-mail: jorge.zabadal@ufrgs.br

\section{APENDICE - Código fonte do método}

Para o problema apresentado no item Aplicação do Método, temos o seguinte código fonte, escrito em linguagem Maple.

$>$ restart:

$>$ with(plots):

$>$ with(stats):

Fonte

$>Q:=-2^{*}\left(-3^{*} a^{\wedge} 2^{*} u^{\wedge} 2+a^{*} b^{*} v^{\wedge} 2+a^{*} c-3^{*} b^{\wedge} 2^{*} v^{\wedge} 2+b^{*} a^{*} u^{\wedge} 2+b^{*} c\right) /\left(a^{*} u^{\wedge} 2+b^{*} v^{\wedge} 2+c\right)^{\wedge} 3$

$>\mathrm{q}:=\operatorname{subs}\left(\mathrm{u}=(\mathrm{w}+\mathrm{wc}) / 2, \mathrm{v}=\mathrm{I}^{*}(\mathrm{wc}-\mathrm{w}) / 2, \mathrm{Q}\right)$ :

Integração do termo fonte.

$>\mathrm{q} 1:=\operatorname{int}(\mathrm{q}, \mathrm{w}) / 4$ :

$>$ f0_1:=int $(\mathrm{q} 1, \mathrm{wc})$ :

$>\mathrm{f} 0 \_2:=\operatorname{subs}\left(\mathrm{w}=\mathrm{u}+\mathrm{I}^{*} \mathrm{v}, \mathrm{wc}=\mathrm{u}-\mathrm{I}^{*} \mathrm{v}, \mathrm{f} 0 \_1\right)$ :

Extração da parte real.

$>$ f0_3:=evalc(Re(f0_2)):

$>\mathrm{f0}:=\operatorname{simplify}\left(\mathrm{f0} \_3\right)$ :

Substituição dos coeficientes.

$>\mathrm{C}:=\operatorname{subs}(\{\mathrm{a}=.00000012, \mathrm{~b}=.000000097, \mathrm{c}=.00173\}, \mathrm{f0})$ :

$>$ plot3d(C,u=-500..500,v=0..500,axes=boxed):

Ajuste das paramétricas.

$>$ restart:

$>$ with(stats):

with(plots):

$>$ pontos: $=\{[133.85,-728.76],[118.98,-490.8],[74.36,-208.22],[0,0],[-74.36,133.85],[59.49,446.18],[609.78,773.37],[$

1308.79,773.37]\}:

$>$ yp:=rhs $\left(\right.$ fit $\left[\right.$ leastsquare $\left.\left[[x, y], y=e^{*} x^{\wedge} 4+a^{*} x^{\wedge} 3+b^{*} x^{\wedge} 2+c^{*} x+d,\{e, a, b, c, d\}\right]\right]([[-500,-300,-100,0,100,300,400,500],[-$

728.76,-490.8,-208.22,0,133.85,446.18,773.37,773.3]])):

$>$ ypu: $=\operatorname{subs}\left(\mathrm{x}=\mathrm{u}+\mathrm{I}^{*} \mathrm{v}, \mathrm{yp}\right)$ :

$>$ xp: $=\operatorname{rhs}\left(\right.$ fit $\left[\right.$ leastsquare $\left.\left[[x, y], y=e^{*} x^{\wedge} 4+a^{*} x^{\wedge} 3+b^{*} x^{\wedge} 2+c^{*} x+d,\{e, a, b, c, d\}\right]\right]([[-500,-300,-100,0,100,300,400,500],[133.8$

$5,118.98,74.36,0,-74.36,59.49,609.78,1308.79]]))$ :

$>\mathrm{xpu}:=\operatorname{subs}\left(\mathrm{x}=\mathrm{u}+\mathrm{I}^{*} \mathrm{v}, \mathrm{xp}\right)$ :

Função transformação.

$>\mathrm{z}$ :=evalc $\left(\mathrm{xpu}+\mathrm{I}^{*} \mathrm{ypu}\right)$ :

Determinação dos pontos no plano $z$.

$>\mathrm{x}:=\operatorname{evalc}(\operatorname{Re}(\mathrm{z}))$ :

$>\mathrm{y}:=\operatorname{evalc}(\operatorname{Im}(\mathrm{z}))$ :

$>$ for $\mathrm{u}$ from -600 by 300 to 600 do

for $\mathrm{v}$ from 1 by 300 to 600 do

$\operatorname{Print}(\mathrm{x}) ; \operatorname{print}(\mathrm{y}) ; \operatorname{print}(\mathrm{f} 0)$ : od:

od: 